International Journal of Electrical Engineering and Technology (IJEET)

Volume 12, Issue 5, May 2021, pp. 109-115, Article ID: IJEET_12_05_010

Available online at https://iaeme.com/Home/issue/IJEET? Volume $=12 \&$ Issue $=5$

ISSN Print: 0976-6545 and ISSN Online: 0976-6553

DOI: 10.34218/IJEET.12.5.2021.010

(C) IAEME Publication

Scopus Indexed

\title{
A SMART PARKING SYSTEM USING M2M COMMUNICATION
}

\author{
D Sony \\ Department of Electronics and Communication Engineering \\ Chaitanya Bharathi Institute of Technology, Telangana, India \\ N Krishna Sandeep Reddy \\ Department of Electronics and Communication Engineering \\ Chaitanya Bharathi Institute of Technology, Telangana, India

\section{R V Krishna Sathvik} \\ Department of Electronics and Communication Engineering \\ Chaitanya Bharathi Institute of Technology, Telangana, India

\section{Palle Vivek Goud} \\ Department of Electronics and Communication Engineering \\ Chaitanya Bharathi Institute of Technology, Telangana, India
}

\begin{abstract}
With an increase in the population of the vehicles in metropolitan cities, road congestion is the major problem that is being faced and the time, efforts and fuel are wasted in search of the availability of the free space in a specified parking area. Growth of Internet of Things (IoT) has paved way for integration of mobile devices, wireless communicationtechnologies and mobile applications. To resolves these issues, an IoT based cloud integrated Smart Parking system with mobile application is being proposed. The whole system is based on machine-to-machine(M2M) communication in IoT. The proposed Smart Parking system consists of an on-site deployment of an IoT module that is used to monitor and signalize the state of availability of each single parking space. Pervasive presence of smart phone encourages users to prefer mobile application-based solutions.
\end{abstract}

Key words: IoT, Parking System, M2M communication, population

Cite this Article: D Sony, N Krishna Sandeep Reddy, R V Krishna Sathvik and Palle Vivek Goud, A Smart Parking System using M2M Communication, International Journal of Electrical Engineering and Technology (IJEET), 12(5), 2021, pp. 109-115. https://iaeme.com/Home/issue/IJEET?Volume=12\&Issue $=5$ 


\section{INTRODUCTION}

In our daily lives, we all have experienced frustration due to unavailability of parking spaces in desired places or the difficulty in finding a safe and suitable parking spot. In a recent research it has been found that a driver takes nearly 8 minutes to park his vehicle because he spends more time in searching for the parking lot. This searching leads to about 30-40\% of traffic congestion [1] [2]. This issue has created a concern among several sectors and the need to come up with efficient technology enabled solutions has risen. The Internet of Things (IoT) [3] permeates with the world of parking to streamline processes that deliver intelligent parking solutions, which extend and manage parking inventories. The IoT describes the network of physical objects - a.k.a. "things"- that are embedded with sensors, software, and other technologies for the purpose of connecting and exchanging data with other devices and systems over the Internet [4]. In this context, IoT uses wireless sensor networks to connect physical parking space infrastructures with information and communication technologies, where cloudbased smart management services are provided.

Currently, there is no organized method for finding parking slots in most of the areas. The driver has to manually go through the area and find a suitable spot for parking. This process takes time and effort and may lead to the worst case of failing to find any parking space if the driver is driving in a city with high vehicle density. Recent research has used vehicle-to-vehicle and vehicle-to-infrastructure interaction with the support of various wireless network technologies. This study aimed at enabling a user to book a parking slot in a desired location by providing information about vacant parking spots over a user-friendly web page. The user can access the web page using any smart gadget such as a smart phone, laptop, or a computer. However, this idea has not been implemented effectively till date. The IoT has created a revolution in many fields in life as well as in smart-parking system (SPS) technology [5].

The present study proposes and develops an effective cloud based SPS solution based on the IoT. Our system builds each parking spot as an IoT network, and the data that include the information from sensors, number of slots and their availability will be transferred to the cloud. The SPS is based on several innovative technologies and can automatically monitor and manage car parks.

A smart parking system with a reservation policy provides many advantages for both the users (i.e., drivers) and parkingareas controllers ${ }^{[6]}$. A parking reservation system allows users to book their parking spots prior to their arrival throughthe use of advanced communication technologies such as a web interface. A smart parking reservation system has majoradvantages over the traditional Parking Guidance ${ }^{[7]}$ and Information (PGI) systems. The users are continuously informed about the availability of the parking slot through a web page. In this paper, we propose an intelligent parking system which is easy to access by users and enables them to book a slot using the web page that we have developed and programmed to display the real time information about the availability of parking slots in the desired locations. In this system, the data is collected from the sensors and through analyzing and processing, the output is obtained. This systemalso makes sure that parking slot is secure.

\section{METHODOLOGY}

In the everyday life, everyone would have some kind of work that needs to be attended to. In some cases, these workscan be done from home while others require physical presence. So, the tasks requiring presence will mean for that person to utilize a vehicle, whether it's public transport or own. People travelling by their own vehicles always require space to park while addressing those tasks. Few places may have a dedicated parking space, but others do not always haveone. Also, the ones with a space may not always be available. To solve this crisis, this webapp can be utilized. 
Firstly, a user goes to our web application where he/she willbe asked to login. If they are a new user, they can Sign up through a form they are redirected to on the click of new user.Upon successful signup, a message "Registered successfully" is displayed to the user. If it fails, then a message "User already exists" is displayed.

The signup form information is stored in a database created in MongoDB ${ }^{[8]}$. It stores data such as the Name, Username, Email, Password. Storing password of a user is always a riskas it may be prone to hacking, in order to avoid that problem, password hashing is done which ensures that the password isstored as an encrypted one.

A database is used in order to validate a user's login i.e., when a registered user is logging in, the username and password they are using should match the ones they had givenwhile signing up. Also, if a new user while signing up uses analready in use username, then also a message saying that "User already exists" is displayed. These validations are veryimportant to avoid misuse of a user account and also avoid duplicates of an account with the same username.

After logging in, the user is displayed two places. Based on which place they want to park their vehicle; they select one of those shown places. When the location is selected, the useris redirected to a page where the parking slots availability is displayed. As shown in Fig.1, the ultrasonic sensor connected to the Raspberry Pi, detects whether any vehicle is parked in that slot every 5 seconds ${ }^{[9]}$. This information is sent to Firebase where it gets updated in the Realtime database.

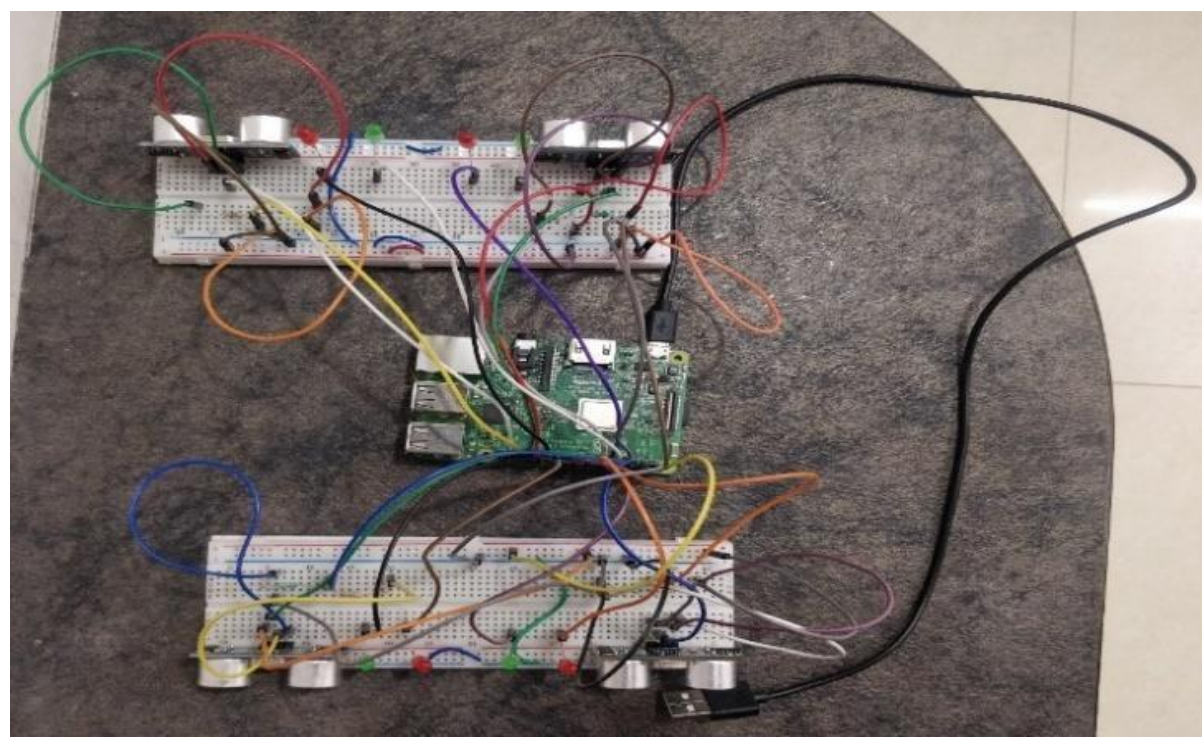

Figure 1 Hardware Implementation

The status of slot from the Realtime Database is immediatelyupdated in the web application for the user to view and decidethe slot they want to reserve. Depending on the vacancy of the parking slot, the user can book the slot which is vacant. Ifthe slot isn't available, the user can look for slots in the other parking space. When a vacant slot is booked by the user, they are redirected to a page displaying the message that the booking has been successful. The user can then go park at thatslot without any hassle. For the people who do not use the webapplication, the LED's present on the outside of the slot indicate the availability. This helps when a person unknowingly parks their vehicle without knowing that it has been reserved by someone else. 


\section{RESULTS}

This paper has developed a raspberry pi based advanced/smart vehicle parking system. As the user logs into the web application, his/her data is updated in MongoDB as shown in Fig. 2. The user data stored involves the username, email and password.

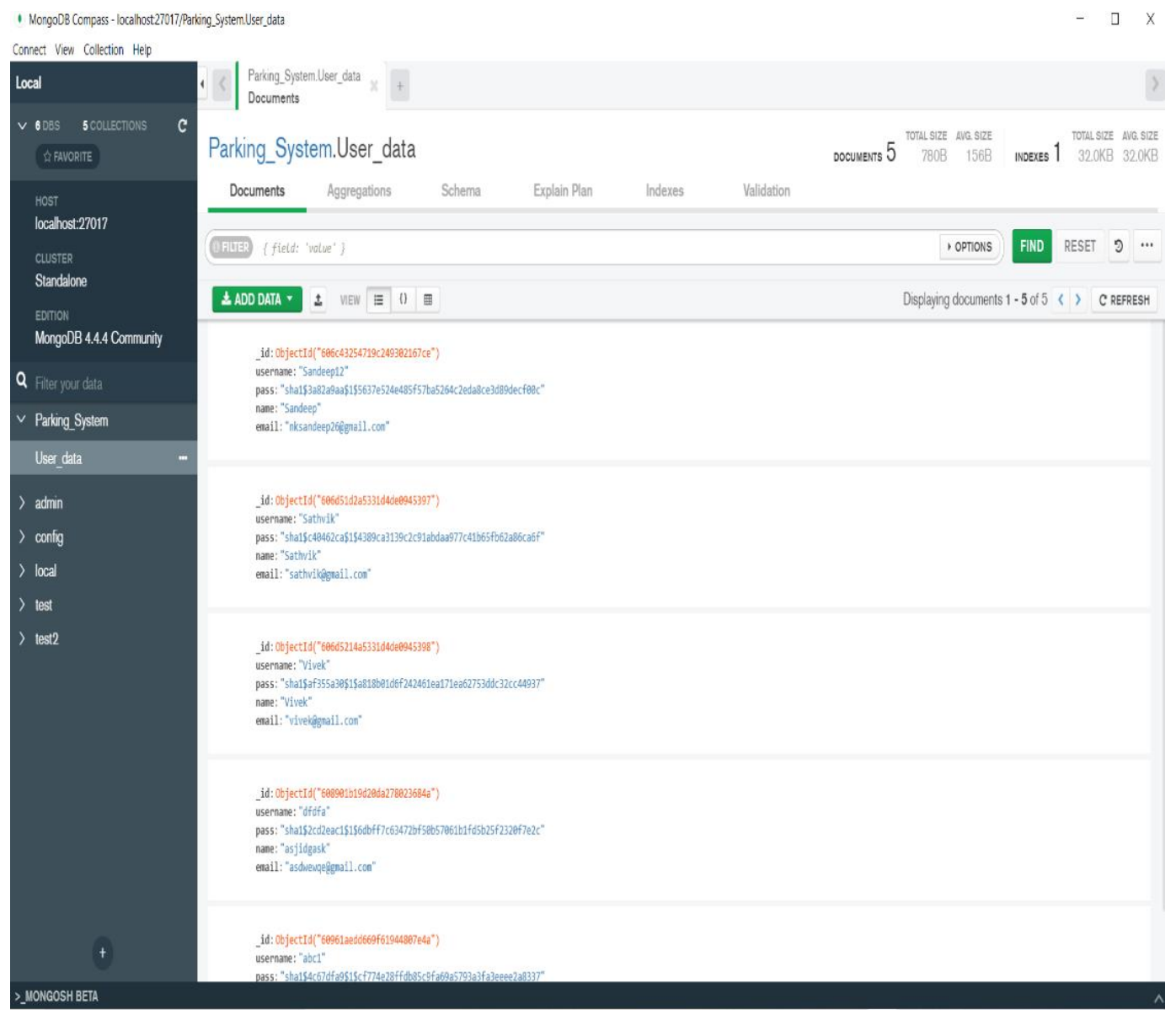

Figure 2. User Database in MongoDB

To avoid the duplication of the data that is being updated in the database, the user's data is validated for every new registration using NodeJS. This is depicted in Fig. 3.

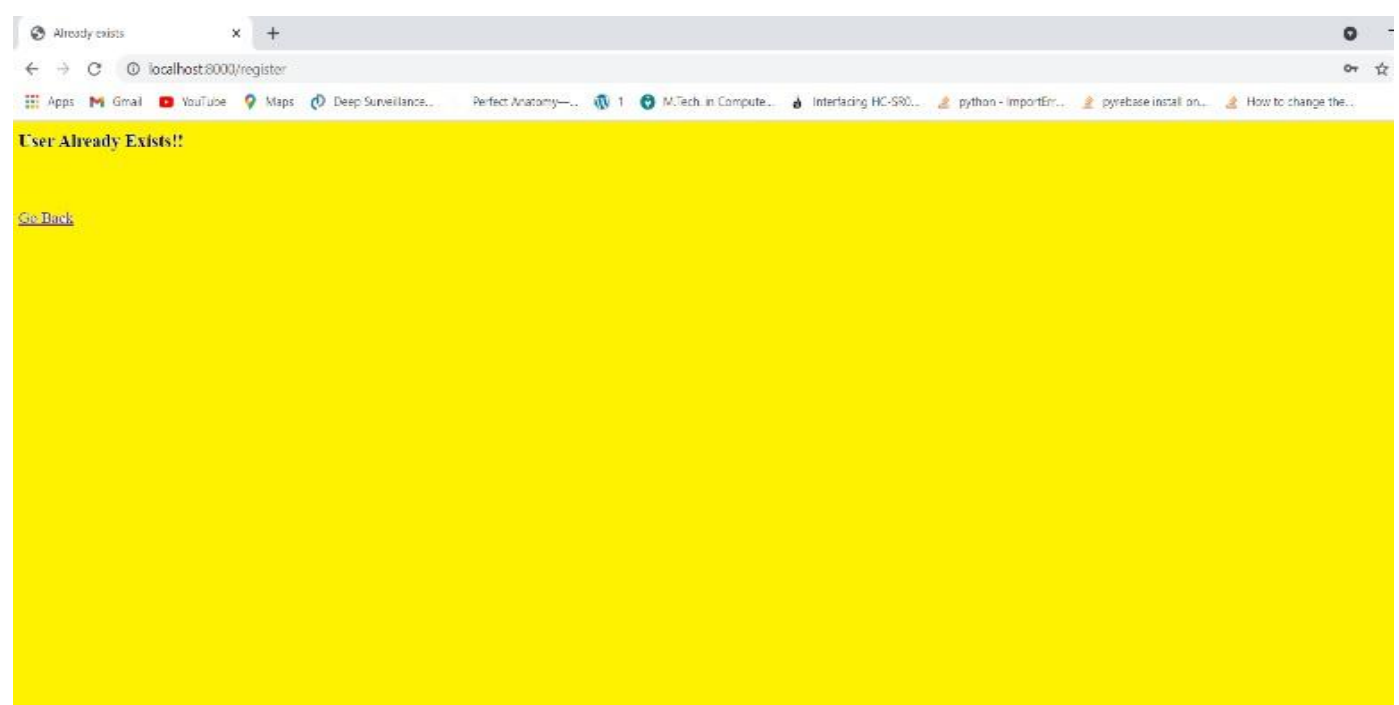

Figure 3. Validation of user to avoid duplication 
As per customer's requirement a parking slot is being selected. All the slots are updated in real time, using Firebaseas shown in Fig. 4.

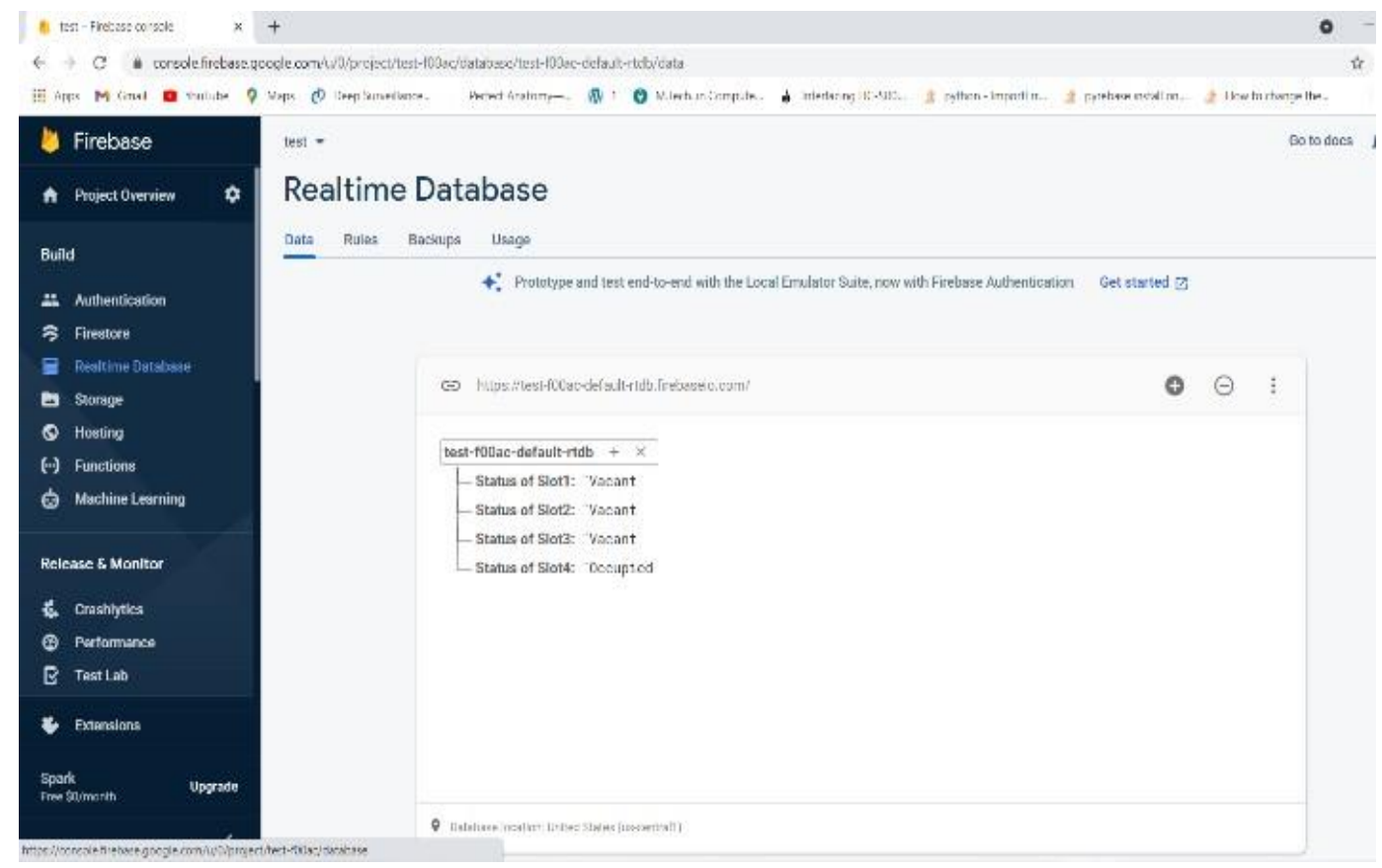

Figure 4. Realtime Database in Firebase

The ultrasonic sensors used in this projectat the parking slot are used to detect the vacant slots and prompts the user to book the available slot in the web application as shown in Fig. 5.

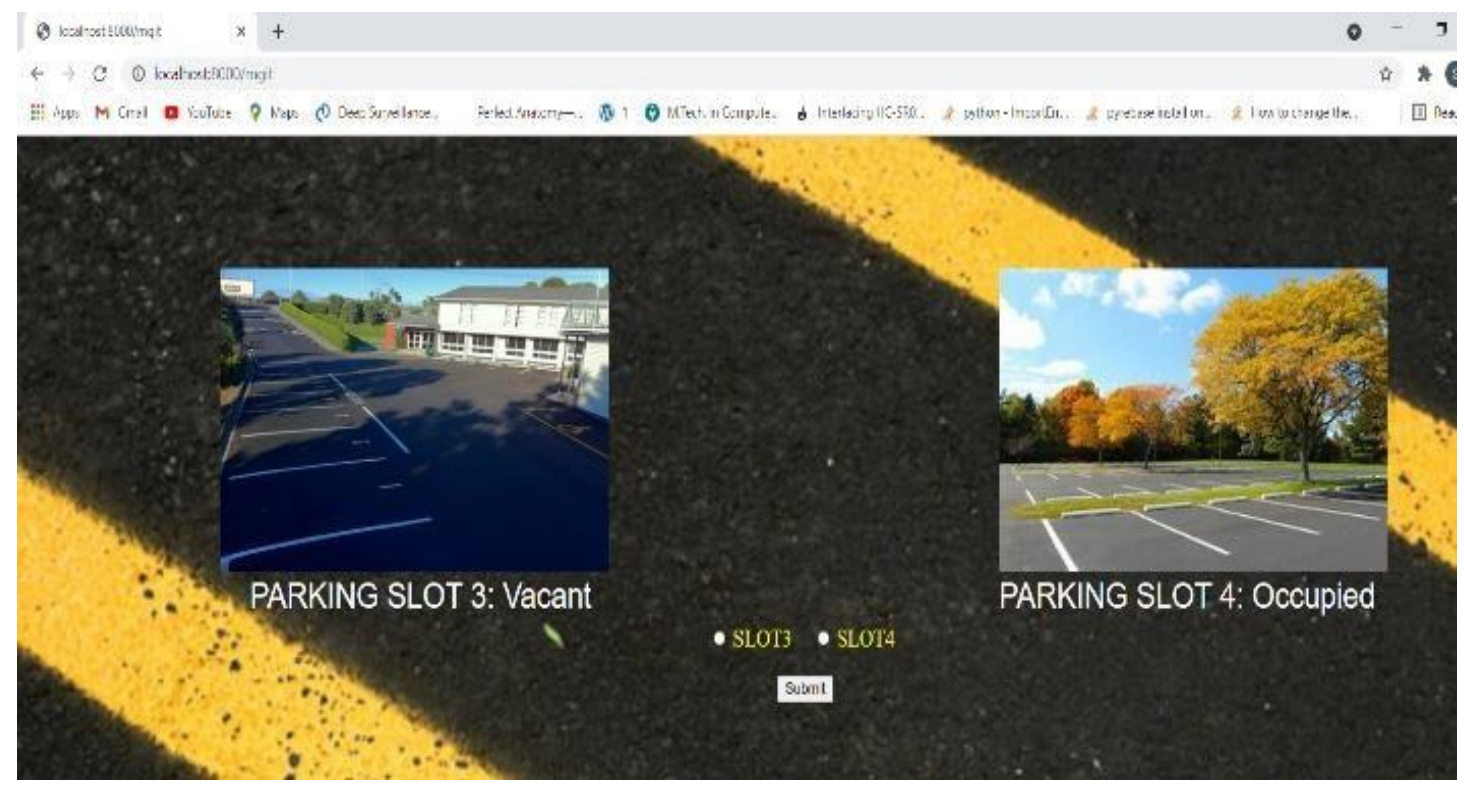

Figure 5. User Interface for Slot Availability

As soon as the booking is donebased on the availability, a confirmation message is displayed to user in the webpage. This is shown in Fig. 6. 
A Smart Parking System using M2M Communication

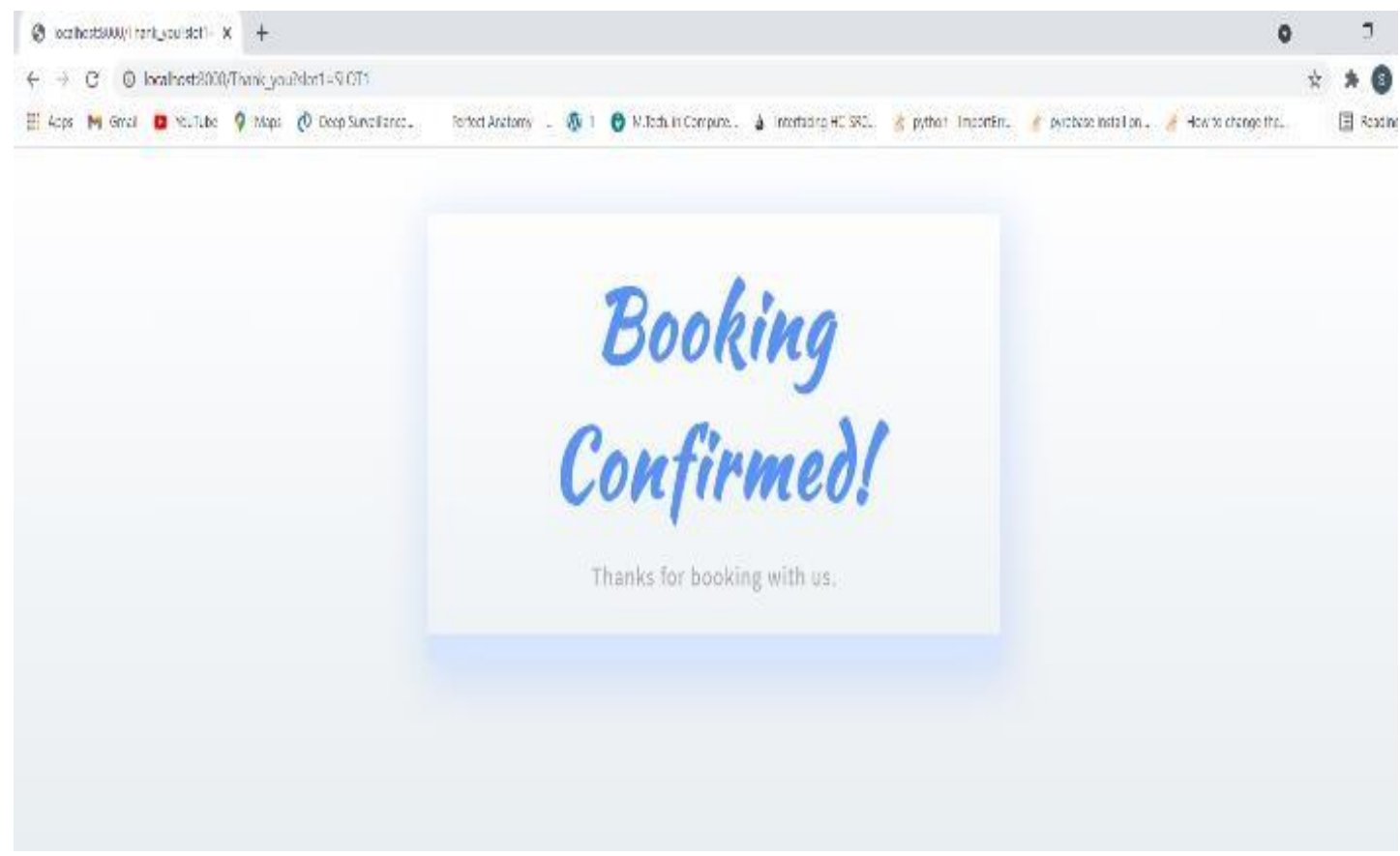

Figure 6. Confirmation of Booking Slot

LED indicators interfaced along with the sensor circuitry help the people who do not use the web application in knowing whether a particular spot is reserved or not. This is indicated in Fig. 7.

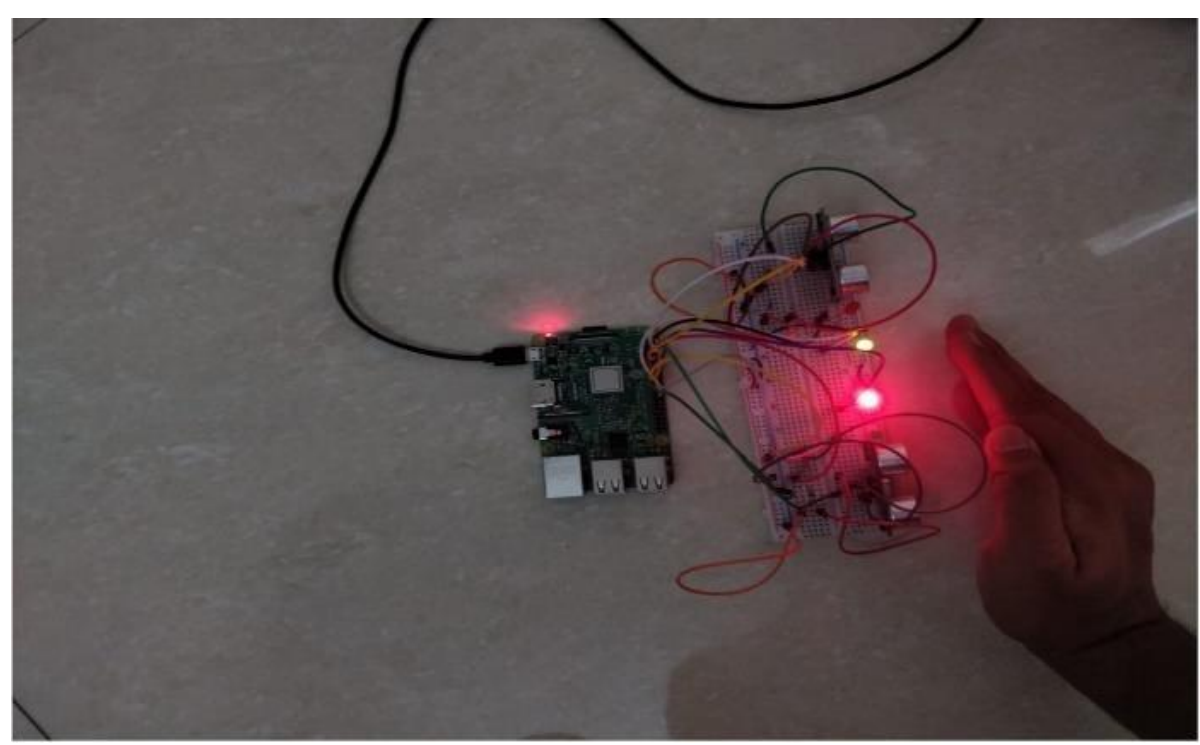

Figure 7. LED indicators for indication of slot status

\section{CONCLUSION}

As the population is increasing, the number of cars is also increasing rapidly. This makes it quite a challenge to find a parking space. Issues like traffic congestion, unnecessary fuel consumption etc. are rising in search of a parking space. TheSmart Parking System proposed in this paper has been implemented using M2M communication, helps in the reduction of above discussed issues. Also, with the designed web application, it makes it easier for the users to reserve a parking space at any place they want.

To make the system more viable and efficient in the coming future, an automated billing system can be added. Along with that, to increase the security measures, vehicle number or driver face recognition can be included. 


\section{REFERENCES}

[1] L. Mainetti, L. Palano, L. Patrono, M. L. Stefanizzi, and R. Vergallo, "Integration of RFID and WSN technologies in a smart parking system", in IEEE 22nd International Conference on SoftCOM, September 2014

[2] D. J. Bonde, R. S. Shende, K. S. Gaikwad, A. S. Kedari, and A. U. Bhokre, "Automated car parking system commanded by Android application", in IEEE International Conference on Computer Communication and Informatics, January 2014

[3] Baratam. M Kumar Gandhi, M. Kameswara Rao, "IoT based Car Parking Management System for Smart Cities", Indian Journal of Science And Technology, May 2016.

[4] Om Prakash Yadav, Dr. R.P. Singh, "Smart Parking System usingM2M communication in IoT", JETIR February 2019.

[5] L. Lambrinos and L. Dosis, "DisAssist: An Internet of Things and mobile communications platform for disabled parking space management", in IEEE Global Communications Conference (GLOBECOM), December 2013.

[6] R. E. Barone, T. Giuffrè, S. M. Siniscalchi, M. A. Morgano, and

[7] G. Tesoriere, "Architecture for parking management in smart cities", Intelligent Transport Systems (IET), ResearchGate January 2013.

[8] R. Lu, X. Lin, H. Zhu, and X. S. Shen, "An Intelligent Secure andPrivacy-Preserving Parking Scheme Using Vehicular Communications", in IEEE Transactions on Vehicular Technology, 2009.

[9] Akash Gupta, Priyansh Rastogi, Shaurya Jain, "Smart Parking System using Cloud based Computation and Raspberry Pi" in IEEE 2nd International Conference on I-SMAC,2018.

[10] C. Shiyao, W. Ming, L. Chen, and R. Na, "The research and implement of the intelligent parking reservation management system based on ZigBee technology", in IEEE $6^{\text {th }}$ International Conference on Measuring Technology and Mechatronics Automation (ICMTMA), January 2014.

[11] C. W. Hsu, M. H. Shih, H. Y. Huang, Y. C. Shiue, and S. C. Huang, "Verification of smart guiding system to search for parking space via DSRC communication", in IEEE 12th International Conference on ITS Telecommunications, February 2013 